\title{
A MAXIMUM PRINCIPLE FOR STOCHASTIC OPTIMAL CONTROL WITH TERMINAL STATE CONSTRAINTS, AND ITS APPLICATIONS*
}

\author{
SHAOLIN $\mathrm{JI}^{\dagger}$ AND XUN YU ZHOU ${ }^{\ddagger}$
}

\begin{abstract}
This paper is concerned with a stochastic optimal control problem where the controlled system is described by a forward-backward stochastic differential equation (FBSDE), while the forward state is constrained in a convex set at the terminal time. An equivalent backward control problem is introduced. By using Ekeland's variational principle, a stochastic maximum principle is obtained. Applications to state constrained stochastic linear-quadratic control models and a recursive utility optimization problem are investigated.
\end{abstract}

Keywords: Forward-backward stochastic differential equation (FBSDE); state constraints; Ekeland's variational principle; maximum principle; recursive utility; linear-quadratic control

1. Introduction. The modern optimal stochastic control theory has been well developed since early 1960s, along the lines of Pontryagin's maximum principle (MP), Bellman's dynamic programming (DP) and Kalman's linear-quadratic (LQ) control. A recent systematic account on the theory can be found in [13].

Sophisticated and rich as it may be, the existing stochastic control theory could only handle pathetically limited problems. In particular, in general it has to assume that there is no sample-wise constraint imposed on the state (a sample-wise constraint requires that the state at certain time or at all times be in a prescribed set with probability 1), whereas the existence of such a constraint in reality is more a rule than an exception.

The reason that the current theory is generally incapable of solving the state constrained stochastic control is that the two main approaches, MP and DP, fall apart with the additional constraints. Specifically, a sample-wise state constraint essentially introduces infinitely many additional constraints compared with a deterministic state constrained control problem. This is also why there is indeed MP derived for the latter where a so-called transversality condition is introduced [2], whereas its stochastic counterpart is simply non-existent in literature. As for the DP approach, even in the deterministic setting a state constraint may cause non-continuity of the value function

\footnotetext{
* Dedicated to Tyrone Duncan on the occasion of his 65th birthday.

${ }^{\dagger}$ School of Mathematics \& System Sciences, Shandong University, Jinan 250100, China. Tel.: 86-0531-88364100, fax: 86-0531-88564100, E-mail: jsl@sdu.edu.cn.

${ }^{\ddagger}$ Department of Systems Engineering and Engineering Management, The Chinese University of Hong Kong, Shatin, Hong Kong. Tel.: 852-2609-8320, fax: 852-2603-5505, email: xyzhou@se.cuhk.edu.hk. Supported by the RGC Earmarked Grants \#418606 and CUHK4175/03E.
} 
[1], leading to the non-applicability of the DP.

This paper attacks this open problem of stochastic control with state constraints. Specifically, the controlled system under consideration is described by a forwardbackward stochastic differential equation (FBSDE) which includes the usual forward $\mathrm{SDE}$ as a special case, while the forward state is constrained in a convex set at the terminal time. Such a general problem without state constraints was introduced and studied in [10]. However, as discussed above the presence of a state constraint poses great difficulty. The main idea of this paper to overcome the difficulty is inspired by some recently developed approaches in finance, i.e., terminal perturbation method, which is used to study the recursive utility optimization problem ([7]) and dual approach in solving a continuous-time mean-variance portfolio selection model ([3]).

El Karoui, Peng and Quenez [7] consider a portfolio-consumption model where the objective is to optimize the recursive utility of consumption and terminal wealth. They introduce the terminal perturbation method to solve this problem: they first give a backward formulation of this optimization problem in which the terminal wealth is regarded as the "control variable", and then perturb the terminal wealth to obtain a necessary condition for the optimal terminal wealth. On the other hand, the dual approach is employed by Bielecki et al. [3] to solve a continuous-time portfolio selection model with nonnegativity constraint on the wealth process. This approach was first introduced for discrete-time models by Pliska [11], [12]. It includes two steps: the first step is to compute the optimal terminal wealth, and the second one is to obtain the portfolio replicating the obtained optimal terminal wealth.

Appealing to these two approaches, we first re-formulate FBSDE controlled system as a purely backward system by taking the terminal condition of the forward state as a "control variable". In this case the original initial condition of the forward state becomes an additional constraint. Different from the Lagrange multiplier method used in [3] and [7] to deal with this additional constraint, we apply Ekeland's variational principle instead to derive a stochastic maximum principle which characterizes the optimal terminal state. This technique is introduced in [8] and [9] to tackle the non-convex state equations.

We also give two applications of the established stochastic maximum principle. The first one is a stochastic linear-quadratic (LQ) optimal control problem with terminal state constraints, and the second one is a recursive utility optimization problem considered in El Karoui, Peng and Quenez [7]. It is worth pointing out that our method makes no convexity assumption on the coefficients of the nonlinear wealth equation, as opposed to [7].

This paper is organized as follows. In section 2, we formulate the model under consideration as well as its equivalent backward formulation. Applying terminal 
perturbation method and Ekeland's variational principle we obtain a stochastic maximum principle in section 3. In section 4 , we study its applications to stochastic LQ control and recursive utility optimization problem. Finally, section 5 closes the paper with some concluding remarks.

2. Problem Formulation. Let $W(\cdot)=\left(W_{1}(\cdot), \ldots, W_{d}(\cdot)\right)^{\prime}$ be a standard $d$ dimensional Brownian motion defined on a complete probability space $(\Omega, \mathcal{F}, P)$. The information structure is given by a filtration $F=\left\{\mathcal{F}_{t}\right\}_{0 \leq t \leq T}$, which is generated by $W(\cdot)$ and augmented by all the $P$-null sets. For any given Euclidean space $H$, we denote by $\langle\cdot, \cdot\rangle$ (resp. $|\cdot|)$ the scalar product (resp. norm) of $H$. Let $M^{2}(0, T ; H)$ denote the space of all $\mathcal{F}_{t}$-progressively measurable processes $x(\cdot)$ with values in $H$ such that

$$
E \int_{0}^{T}|x(t)|^{2} d t<\infty .
$$

We denote by $L\left(R^{n} ; R^{m}\right)$ the Euclidean space of all $m \times n$ matrices.

2.1. Classical formulation. Consider the following stochastic optimal control problem for a forward-backward system, which was formulated in [10].

Let

$$
\mathcal{U}_{a d} \equiv\left\{u(\cdot) \mid u(\cdot) \in M^{2}\left(0, T ; L\left(R^{d} ; R^{n}\right)\right)\right\},
$$

whose elements are called admissible controls. For any given admissible control $u(\cdot)$, we consider the following forward and backward SDE (FBSDE):

$$
\left\{\begin{array}{l}
d x(t)=b(x(t), u(t), t) d t+\sigma(x(t), u(t), t) d W(t), \\
x(0)=a \\
-d y(t)=\bar{g}(x(t), y(t), z(t), u(t), t) d t-z(t) d W(t), \\
y(T)=h(x(T))
\end{array}\right.
$$

where $a \in R^{n}$ is given,

$$
\begin{aligned}
b & : R^{n} \times L\left(R^{d} ; R^{n}\right) \times[0, T] \rightarrow R^{n}, \\
\sigma & : R^{n} \times L\left(R^{d} ; R^{n}\right) \times[0, T] \rightarrow L\left(R^{d} ; R^{n}\right), \\
\bar{g} & : R^{n} \times R^{m} \times L\left(R^{d} ; R^{m}\right) \times L\left(R^{d} ; R^{n}\right) \times[0, T] \rightarrow R^{m}, \\
h & : R^{n} \rightarrow R^{m} .
\end{aligned}
$$

Define the following cost function:

$$
J(u(\cdot))=E\left[\int_{0}^{T} \bar{l}(x(t), y(t), z(t), u(t), t) d t+\phi(x(T))+\gamma(y(0))\right]
$$

where

$$
\begin{aligned}
\bar{l} & : R^{n} \times R^{m} \times L\left(R^{d} ; R^{m}\right) \times L\left(R^{d} ; R^{n}\right) \times[0, T] \rightarrow R, \\
\phi & : R^{n} \rightarrow R, \\
\gamma & : R^{m} \rightarrow R .
\end{aligned}
$$


We assume:

(H1) $b, \sigma, \bar{g}, h, \bar{l}, \phi$ and $\gamma$ are continuous in their arguments and continuously differentiable in $(x, y, z, u)$;

(H2) the derivatives of $b, \sigma, \bar{g}$ and $h$ in $(x, y, z, u)$ are bounded;

(H3) the derivatives of $\bar{l}$ in $(x, y, z, u)$ are bounded by $C(1+|x|+|y|+|z|$ $+|u|)$, and the derivatives of $\phi$ and $\gamma$ in $x$ are bounded by $C(1+|x|)$.

Then, for given $u(\cdot) \in \mathcal{U}_{a d}$, there exists a unique triple

$$
(x(\cdot), y(\cdot), z(\cdot)) \in M^{2}\left(0, T ; R^{n}\right) \times M^{2}\left(0, T ; R^{m}\right) \times M^{2}\left(0, T ; L\left(R^{d} ; R^{m}\right)\right)
$$

which solves $(2.1)$ (see $[6,13])$. The corresponding solution $(x(\cdot), y(\cdot), z(\cdot))$ is the state variable for our control problem.

An essential different (and difficult) feature compared with [10] is that we consider an additional convex constraint on the terminal state variable $x(\cdot)$ by

$$
x(T) \in K, \quad \text { a.s. }
$$

where $K$ is a given nonempty convex subset in $R^{n}$.

In summary, our stochastic control problem is

$$
\begin{array}{ll}
\text { Minimize } & J(u(\cdot)) \\
\text { subject to } & u(\cdot) \in \mathcal{U}_{a d} ; \quad x(T) \in K, \quad \text { a.s. }
\end{array}
$$

Remark: Clearly the usual stochastic control problem where the dynamics involve only the forward component $x(\cdot)$ is a special case of $(2.2)$.

2.2. Backward formulation. In this subsection, we give an equivalent backward formulation of the above stochastic optimal control problem (2.2). To do so we need an additional assumption:

(H4) there exists $\alpha>0$ such that $\left|\sigma\left(x, u_{1}, t\right)-\sigma\left(x, u_{2}, t\right)\right| \geq \alpha\left|u_{1}-u_{2}\right|$ for all $x \in R^{n}, t \in[0, T]$ and $u_{1}, u_{2} \in L\left(R^{d} ; R^{n}\right)$.

Note that (H1), (H2) and (H4) imply the mapping

$$
u \rightarrow \sigma(x, u, t)
$$

is a bijection from $L\left(R^{d} ; R^{n}\right)$ onto itself for any $(x, t)$.

Let $q \equiv \sigma(x, u, t)$ and denote the inverse function by $u=\widetilde{\sigma}(x, q, t)$. Then system (2.1) can be rewritten as

$$
\left\{\begin{array}{l}
-d x(t)=f(x(t), q(t), t) d t-q(t) d W(t) \\
x(0)=a \\
-d y(t)=g(x(t), y(t), z(t), q(t), t) d t-z(t) d W(t) \\
y(T)=h(x(T))
\end{array}\right.
$$


where $f(x, q, t) \equiv-b(x, \widetilde{\sigma}(x, q, t), t)$ and $g(x, y, z, q, t)=\bar{g}(x, y, z, \widetilde{\sigma}(x, q, t), t)$.

A key observation that inspires our approach of solving problem (2.2) is that, since $u \rightarrow \sigma(x, u, t)$ is a bijection, $q(\cdot)$ can be regarded as the control; moreover, by the BSDE theory selecting $q(\cdot)$ is equivalent to selecting the terminal value $x(T)$. Hence we introduce the following "controlled" system:

$$
\left\{\begin{array}{l}
-d x(t)=f(x(t), q(t), t) d t-q(t) d W(t) \\
x(T)=\xi \\
-d y(t)=g(x(t), y(t), z(t), q(t), t) d t-z(t) d W(t), \\
y(T)=h(\xi)
\end{array}\right.
$$

where the "control" is the random variable $\xi$ to be chosen from the following set

$$
U=\left\{\left.\xi|E| \xi\right|^{2}<\infty, \xi \in K, \text { a.s. }\right\} .
$$

For each $\xi \in U$, consider the following cost

$$
J(\xi)=E\left[\int_{0}^{T} l(x(t), y(t), z(t), q(t), t) d t+\phi(\xi)+\gamma(y(0))\right]
$$

where $l(x, y, z, q, t)=\bar{l}(x, y, z, \widetilde{\sigma}(x, q, t), t)$.

This gives rise to the following auxiliary optimization problem:

$$
\begin{array}{ll}
\text { Minimize } & J(\xi) \\
\text { subject to } & \xi \in U ; \quad x^{\xi}(0)=a
\end{array}
$$

where $x^{\xi}(0)$ is the solution of $(2.3)$ at time 0 under $\xi$.

It is clear that the original problem (2.2) is equivalent to the auxiliary one (2.4). Hence, hereafter we focus ourselves on solving (2.4). The advantage of doing this is that, since $\xi$ now is the control variable, the state constraint in (2.2) becomes a control constraint in (2.4), whereas it is well known in control theory that a control constraint is much easier to deal with than a state constraint. There is, nonetheless, a cost of doing so; that is the original initial condition $x(0)=a$ now becomes a constraint, as shown in (2.4).

From now on, we denote the solution of (2.3) by $\left(x^{\xi}(\cdot), y^{\xi}(\cdot), q^{\xi}(\cdot), z^{\xi}(\cdot)\right)$, whenever necessary, to show the dependence on $\xi$. We also denote $x^{\xi}(0)$ and $y^{\xi}(0)$ by $x_{0}^{\xi}$ and $y_{0}^{\xi}$ respectively. Finally, it is easy to check that $f, g$ and $l$ satisfy similar conditions in Assumptions (H1)-(H3).

Definition 2.1. A random variable $\xi \in U$ is called feasible for given a $\in R^{n}$, if the solution of (2.3) satisfies $x_{0}^{\xi}=a$. We shall denote by $\mathcal{N}(a)$ the set of all feasible $\xi$ 's for any given $a$.

A feasible $\xi^{0}$ is called optimal if it attains the maximum of $J(\xi)$ over $\mathcal{N}(a)$. The aim of this paper is to obtain a characterization of $\xi^{0}$, i.e., a stochastic maximum 
principle. Once $\xi^{0}$ is determined, we can obtain the optimal control $u^{0}(\cdot)$ by solving (2.3).

3. Stochastic Maximum Principle. Applying Ekeland's variational principle, we derive maximum principle (necessary condition for optimality) for the optimization problem (2.4) in this section. To ease the presentation we only study the case where $l(x, y, z, q, t)=0$ and $\phi(x)=0$ in subsections 3.1-3.4, and then present the results for the general case in subsection 3.4.

3.1. Variational equation. For $\xi^{1}, \xi^{2} \in U$, define a metric in $U$ by

$$
d\left(\xi^{1}, \xi^{2}\right) \triangleq\left(E\left|\xi^{1}-\xi^{2}\right|^{2}\right)^{\frac{1}{2}}
$$

It is obvious that $(U, d(\cdot, \cdot))$ is a complete metric space. Let $\xi^{0}$ be optimal and $\left(x^{0}(\cdot), y^{0}(\cdot), q^{0}(\cdot), z^{0}(\cdot)\right)$ be the corresponding state processes of $(2.3)$. Take an arbitrary $\xi \in U$. Then, for each $0 \leq \rho \leq 1, \xi^{0}+\rho\left(\xi-\xi^{0}\right) \in U$. Let $\left(x^{\rho}(\cdot), y^{\rho}(\cdot), q^{\rho}(\cdot), z^{\rho}(\cdot)\right)$ be the state processes of (2.3) associated with $\xi^{0}+\rho\left(\xi-\xi^{0}\right)$. To derive the first-order necessary condition in terms of small $\rho$, we let $(\hat{x}(\cdot), \hat{y}(\cdot), \hat{q}(\cdot), \hat{z}(\cdot))$ be the solution of the following system of BSDEs:

$$
\left\{\begin{aligned}
-d \hat{x}(t) & =\left[f_{x}^{0}(t) \hat{x}(t)+f_{q}^{0}(t) \hat{q}(t)\right] d t-\hat{q}(t) d W(t), \\
\hat{x}(T) & =\xi-\xi^{0} \\
-d \hat{y}(t) & =\left[g_{x}^{0}(t) \hat{x}(t)+g_{y}^{0}(t) \hat{y}(t)+g_{z}^{0}(t) \hat{z}(t)+g_{q}^{0}(t) \hat{q}(t)\right] d t-\hat{z}(t) d W(t), \\
\hat{y}(T) & =h_{x}\left(\xi^{0}\right)\left(\xi-\xi^{0}\right)
\end{aligned}\right.
$$

where $f_{x}^{0}(t)=f_{x}\left(x^{0}(t), q^{0}(t), t\right), f_{q}^{0}(t)=f_{q}\left(x^{0}(t), q^{0}(t), t\right)$ and $g_{k}^{0}(t)=g_{k}\left(x^{0}(t), y^{0}(t), z^{0}(t), q^{0}(t), t\right)$ for $k=x, y, z, q$. Equation (3.1) is called the variational equation.

Set

$$
\begin{aligned}
& \tilde{x}_{\rho}(t)=\rho^{-1}\left[x_{\rho}(t)-x^{0}(t)\right]-\hat{x}(t), \\
& \tilde{q}_{\rho}(t)=\rho^{-1}\left[q_{\rho}(t)-q^{0}(t)\right]-\hat{q}(t), \\
& \tilde{y}_{\rho}(t)=\rho^{-1}\left[y_{\rho}(t)-y^{0}(t)\right]-\hat{y}(t), \\
& \tilde{z}_{\rho}(t)=\rho^{-1}\left[z_{\rho}(t)-z^{0}(t)\right]-\hat{z}(t) .
\end{aligned}
$$

We have the following convergence results:

Lemma 3.1. Assuming (H1)-(H4), we have

$$
\begin{aligned}
& \lim _{\rho \rightarrow 0} \sup _{0 \leq t \leq T} E\left[\tilde{x}_{\rho}(t)^{2}\right]=0, \\
& \lim _{\rho \rightarrow 0} E\left[\int_{0}^{T}\left|\tilde{q}_{\rho}(t)\right|^{2} d t\right]=0, \\
& \lim _{\rho \rightarrow 0} \sup _{0 \leq t \leq T} E\left[\tilde{y}_{\rho}(t)^{2}\right]=0, \\
& \lim _{\rho \rightarrow 0} E\left[\int_{0}^{T}\left|\tilde{z}_{\rho}(t)\right|^{2} d t\right]=0 .
\end{aligned}
$$


Proof. We only prove the first two equalities, the other two being similar. From (2.3) and (3.1), we have

$$
\left\{\begin{aligned}
-d \tilde{x}_{\rho}(t) & =\rho^{-1}\left[f\left(x_{\rho}(t), q_{\rho}(t), t\right)-f\left(x^{0}(t), q^{0}(t), t\right)-\rho f_{x}^{0}(t) \hat{x}(t)\right. \\
& \left.-\rho f_{q}^{0}(t) \hat{q}(t)\right] d t-\tilde{q}_{\rho}(t) d W(t) \\
\tilde{x}_{\rho}(T) & =0
\end{aligned}\right.
$$

Let

$$
\begin{aligned}
& A^{\rho}(t)=\int_{0}^{1} f_{x}\left(x^{0}(t)+\lambda \rho\left(\hat{x}(t)+\tilde{x}_{\rho}(t)\right), q^{0}(t)+\lambda \rho\left(\hat{q}(t)+\tilde{q}_{\rho}(t)\right), t\right) d \lambda, \\
& B^{\rho}(t)=\int_{0}^{1} f_{q}\left(x^{0}(t)+\lambda \rho\left(\hat{x}(t)+\tilde{x}_{\rho}(t)\right), q^{0}(t)+\lambda \rho\left(\hat{q}(t)+\tilde{q}_{\rho}(t)\right), t\right) d \lambda, \\
& C^{\rho}(t)=\left[A^{\rho}(t)-f_{x}^{0}(t)\right] \hat{x}(t)+\left[B^{\rho}(t)-f_{q}^{0}(t)\right] \hat{q}(t) .
\end{aligned}
$$

Thus

$$
\left\{\begin{array}{l}
-d \tilde{x}_{\rho}(t)=\left(A^{\rho}(t) \tilde{x}_{\rho}(t)+B^{\rho}(t) \tilde{q}_{\rho}(t)+C^{\rho}(t)\right) d t-\tilde{q}_{\rho}(t) d W(t), \\
\tilde{x}_{\rho}(T)=0 .
\end{array}\right.
$$

Using Itô's formula to $\left|\tilde{x}_{\rho}(t)\right|^{2}$ we get

$$
\begin{aligned}
E & \left|\tilde{x}_{\rho}(t)\right|^{2}+E \int_{t}^{T}\left|\tilde{q}_{\rho}(s)\right|^{2} d s \\
& =2 E \int_{t}^{T}\left\langle\tilde{x}_{\rho}(s), A^{\rho}(s) \tilde{x}_{\rho}(s)+B^{\rho}(s) \tilde{q}_{\rho}(s)+C^{\rho}(s)\right\rangle d s \\
& \leq K E \int_{t}^{T}\left|\tilde{x}_{\rho}(s)\right|^{2} d s+\frac{1}{2} E \int_{t}^{T}\left|\tilde{q}_{\rho}(s)\right|^{2} d s+E \int_{t}^{T}\left|C^{\rho}(s)\right|^{2} d s
\end{aligned}
$$

where $K$ is a constant. So

$$
\begin{aligned}
& E\left|\tilde{x}_{\rho}(t)\right|^{2}+\frac{1}{2} E \int_{t}^{T}\left|\tilde{q}_{\rho}(s)\right|^{2} d s \\
& \quad \leq K E \int_{t}^{T}\left|\tilde{x}_{\rho}(s)\right|^{2} d s+E \int_{t}^{T}\left|C^{\rho}(s)\right|^{2} d s .
\end{aligned}
$$

However, the Lebesgue dominated convergence theorem implies

$$
\lim _{\rho \rightarrow 0} E \int_{0}^{T}\left|C^{\rho}(t)\right|^{2} d t=0
$$

Hence, applying Gronwall's inequality, we obtain the result.

3.2. Variational inequality. In this section, we employ Ekeland's variational principle ([5]) to deal with the initial constraint $x_{0}^{\xi}=a$.

Given the optimal $\xi^{0}$, introduce a mapping $F_{\varepsilon}(\cdot): U \rightarrow R$ by

$$
F_{\varepsilon}(\xi)=\left\{\left|x_{0}^{\xi}-a\right|^{2}+\left(\max \left(0, \gamma\left(y_{0}^{\xi}\right)-\gamma\left(y_{0}^{\xi^{0}}\right)+\varepsilon\right)\right)^{2}\right\}^{\frac{1}{2}}
$$


where $a$ is the given initial state constraint and $\varepsilon$ is an arbitrary positive constant. It is easy to check that the mappings $\left|x_{0}^{\xi}-a\right|^{2}$ and $\gamma\left(y_{0}^{\xi}\right)$, both from $U$ to $R$, are continuous functional on $U$.

TheOREm 3.2. We suppose (H1)-(H4). Let $\xi^{0}$ be an optimal solution to (2.4). Then there exist $h_{1} \in R^{n}$ and $h_{0} \in R$ with $h_{0} \geq 0$ and $\left|h_{0}\right|+\left|h_{1}\right| \neq 0$ such that the following variational inequality holds

$$
\left\langle h_{1}, \hat{x}_{0}\right\rangle+h_{0}\left\langle\gamma_{y}\left(y_{0}^{\xi^{\circ}}\right), \hat{y}_{0}\right\rangle \geq 0
$$

where $\left(\hat{x}_{0}, \hat{y}_{0}\right) \equiv(\hat{x}(0), \hat{y}(0))$ is the solution of (3.1) at time 0 .

Proof. It is easy to check that $F_{\varepsilon}(\cdot)$ is continuous on $U$ and

$$
\begin{aligned}
& F_{\varepsilon}\left(\xi^{0}\right)=\varepsilon \\
& F_{\varepsilon}(\xi)>0, \forall \xi \in U ; \\
& F_{\varepsilon}\left(\xi^{0}\right) \leq \inf _{\xi \in U} F_{\varepsilon}(\xi)+\varepsilon .
\end{aligned}
$$

Thus, from Ekeland's variational principle [5], $\exists \xi^{\varepsilon} \in U$ such that

(i) $F_{\varepsilon}\left(\xi^{\varepsilon}\right) \leq F_{\varepsilon}\left(\xi^{0}\right)$;

(ii) $d\left(\xi^{0}, \xi^{\varepsilon}\right) \leq \sqrt{\varepsilon}$;

(iii) $\left.F_{\varepsilon}(\xi)+\sqrt{\varepsilon} d\left(\xi, \xi^{\varepsilon}\right)\right) \geq F_{\varepsilon}\left(\xi^{\varepsilon}\right) \forall \xi \in U$.

For any $\xi \in U$, set $\xi_{\rho}^{\varepsilon}=\xi^{\varepsilon}+\rho\left(\xi-\xi^{\varepsilon}\right), 0 \leq \rho \leq 1$. Let $\left(x_{\rho}^{\varepsilon}(\cdot), y_{\rho}^{\varepsilon}(\cdot), q_{\rho}^{\varepsilon}(\cdot), z_{\rho}^{\varepsilon}(\cdot)\right)$ (resp. $\left.\left(x^{\varepsilon}(\cdot), y^{\varepsilon}(\cdot), q^{\varepsilon}(\cdot), z^{\varepsilon}(\cdot)\right)\right)$ be the solution of (2.3) under $\xi_{\rho}^{\varepsilon}$ (resp. $\xi^{\varepsilon}$ ), and $\left(\hat{x}^{\varepsilon}(\cdot), \hat{y}^{\varepsilon}(\cdot), \hat{q}^{\varepsilon}(\cdot), \hat{z}^{\varepsilon}(\cdot)\right)$ be the solution of (3.1) in which $\xi^{0}$ is substituted by $\xi^{\varepsilon}$.

From (iii) above, we conclude

$$
F_{\varepsilon}\left(\xi_{\rho}^{\varepsilon}\right)-F_{\varepsilon}\left(\xi^{\varepsilon}\right)+\sqrt{\varepsilon} d\left(\xi_{\rho}^{\varepsilon}, \xi^{\varepsilon}\right) \geq 0 .
$$

On the other hand, similarly to Lemma 3.1 we have

$$
\begin{aligned}
& \lim _{\rho \rightarrow 0} \sup _{0 \leq t \leq T} E\left[\rho^{-1}\left(x_{\rho}^{\varepsilon}(t)-x^{\varepsilon}(t)\right)-\hat{x}^{\varepsilon}(t)\right]=0, \\
& \lim _{\rho \rightarrow 0} \sup _{0 \leq t \leq T} E\left[\rho^{-1}\left(y_{\rho}^{\varepsilon}(t)-y^{\varepsilon}(t)\right)-\hat{y}^{\varepsilon}(t)\right]=0 .
\end{aligned}
$$

Thus,

$$
\begin{aligned}
& x_{\rho}^{\varepsilon}(0)-x^{\varepsilon}(0) \equiv x_{0}^{\xi_{\rho}^{\varepsilon}}-x_{0}^{\xi^{\varepsilon}}=\rho \hat{x}_{0}^{\varepsilon}+o(\rho), \\
& y_{\rho}^{\varepsilon}(0)-y^{\varepsilon}(0) \equiv y_{0}^{\xi_{\rho}^{\varepsilon}}-y_{0}^{\xi^{\varepsilon}}=\rho \hat{y}_{0}^{\varepsilon}+o(\rho) .
\end{aligned}
$$


This leads to the following expansions:

$$
\begin{aligned}
& \left|x_{0}^{\xi_{\rho}^{\varepsilon}}-a\right|^{2}-\left|x_{0}^{\xi^{\varepsilon}}-a\right|^{2}=2 \rho\left\langle x_{0}^{\xi^{\varepsilon}}-a, \hat{x}_{0}^{\varepsilon}\right\rangle+o(\rho), \\
& \left|\gamma\left(y_{0}^{\xi_{\rho}^{\varepsilon}}\right)-\gamma\left(y_{0}^{\xi^{o}}\right)+\varepsilon\right|^{2}-\left|\gamma\left(y_{0}^{\xi^{\varepsilon}}\right)-\gamma\left(y_{0}^{\xi^{o}}\right)+\varepsilon\right|^{2} \\
= & 2 \rho\left[\gamma\left(y_{0}^{\xi^{\varepsilon}}\right)-\gamma\left(y_{0}^{\xi^{0}}\right)+\varepsilon\right]\left\langle\gamma_{y}\left(y_{0}^{\xi^{\varepsilon}}\right), \hat{y}_{0}^{\varepsilon}\right\rangle+o(\rho) .
\end{aligned}
$$

Now we consider two cases:

Case 1: There exists $\rho_{0}>0$ such that $\gamma\left(y_{0}^{\xi_{\rho}^{\varepsilon}}\right)-\gamma\left(y_{0}^{\xi^{o}}\right)+\varepsilon>0$ for all $\rho \in\left(0, \rho_{0}\right)$. In this case,

$$
\begin{aligned}
& \lim _{\rho \rightarrow 0} \frac{F_{\varepsilon}\left(\xi_{\rho}^{\varepsilon}\right)-F_{\varepsilon}\left(\xi^{\varepsilon}\right)}{\rho} \\
& =\lim _{\rho \rightarrow 0} \frac{1}{F_{\varepsilon}\left(\xi_{\rho}^{\varepsilon}\right)+F_{\varepsilon}\left(\xi^{\varepsilon}\right)} \frac{F_{\varepsilon}^{2}\left(\xi_{\rho}^{\varepsilon}\right)-F_{\varepsilon}^{2}\left(\xi^{\varepsilon}\right)}{\rho} \\
& =\frac{1}{F_{\varepsilon}\left(\xi^{\varepsilon}\right)}\left\{\left\langle x_{0}^{\xi^{\varepsilon}}-a, \hat{x}_{0}^{\varepsilon}\right\rangle+\left[\gamma\left(y_{0}^{\xi^{\varepsilon}}\right)-\gamma\left(y_{0}^{\xi^{0}}\right)+\varepsilon\right]\left\langle\gamma_{y}\left(y_{0}^{\xi^{\varepsilon}}\right), \hat{y}_{0}^{\varepsilon}\right\rangle\right\} .
\end{aligned}
$$

Dividing (3.4) by $\rho$ and sending $\rho$ to 0 , we obtain

$$
h_{0}^{\varepsilon}\left\langle\gamma_{y}\left(y_{0}^{\xi^{\varepsilon}}\right), \hat{y}_{0}^{\varepsilon}\right\rangle+\left\langle h_{1}^{\varepsilon}, \hat{x}_{0}^{\varepsilon}\right\rangle \geq-\sqrt{\varepsilon}\left[E\left(\left|\xi-\xi^{\varepsilon}\right|^{2}\right)\right]^{\frac{1}{2}}
$$

where

$$
h_{0}^{\varepsilon}=\frac{1}{F_{\varepsilon}\left(\xi^{\varepsilon}\right)}\left[\gamma\left(y_{0}^{\xi^{\varepsilon}}\right)-\gamma\left(y_{0}^{\xi^{0}}\right)+\varepsilon\right], \quad h_{1}^{\varepsilon}=\frac{1}{F_{\varepsilon}\left(\xi^{\varepsilon}\right)}\left[x_{0}^{\xi^{\varepsilon}}-a\right] .
$$

Case 2: There exists a positive sequence $\left\{\rho_{n}\right\}$ satisfying $\rho_{n} \rightarrow 0$ such that $\gamma\left(y_{0}^{\xi_{\rho_{n}}^{\varepsilon}}\right)-\gamma\left(y_{0}^{\xi^{o}}\right)+\varepsilon \leq 0$. In this case, by its definition $F_{\varepsilon}\left(\xi_{\rho_{n}}^{\varepsilon}\right)=\left\{\left|x_{0}^{\xi_{\rho_{n}}^{\varepsilon}}-a\right|^{2}\right\}^{\frac{1}{2}}$ for sufficiently large $n$. Since $F_{\varepsilon}(\cdot)$ is continuous, we conclude $F_{\varepsilon}\left(\xi^{\varepsilon}\right)=\left\{\left|x_{0}^{\xi^{\varepsilon}}-a\right|^{2}\right\}^{\frac{1}{2}}$.

Now,

$$
\begin{aligned}
& \lim _{n \rightarrow \infty} \frac{F_{\varepsilon}\left(\xi_{\rho_{n}}^{\varepsilon}\right)-F_{\varepsilon}\left(\xi^{\varepsilon}\right)}{\rho_{n}} \\
& =\lim _{n \rightarrow \infty} \frac{1}{F_{\varepsilon}\left(\xi_{\rho_{n}}^{\varepsilon}\right)+F_{\varepsilon}\left(\xi^{\varepsilon}\right)} \frac{F_{\varepsilon}^{2}\left(\xi_{\rho_{n}}^{\varepsilon}\right)-F_{\varepsilon}^{2}\left(\xi^{\varepsilon}\right)}{\rho_{n}} \\
& =\frac{\left\langle x_{0}^{\xi^{\varepsilon}}-a, \hat{x}_{0}^{\varepsilon}\right\rangle}{F_{\varepsilon}\left(\xi^{\varepsilon}\right)} .
\end{aligned}
$$

Similar to Case 1 we derive

$$
\left\langle h_{1}^{\varepsilon}, \hat{x}_{0}^{\varepsilon}\right\rangle \geq-\sqrt{\varepsilon}\left[E\left(\left|\xi-\xi^{\varepsilon}\right|^{2}\right)\right]^{\frac{1}{2}}
$$

where

$$
h_{0}^{\varepsilon}=0, \quad h_{1}^{\varepsilon}=\frac{x_{0}^{\xi^{\varepsilon}}-a}{F_{\varepsilon}\left(\xi^{\varepsilon}\right)} .
$$


In summary, for both cases, we have $h_{0}^{\varepsilon} \geq 0$, and

$$
\left|h_{0}^{\varepsilon}\right|^{2}+\left|h_{1}^{\varepsilon}\right|^{2}=1
$$

by the definition of $F_{\varepsilon}(\cdot)$. Then there exists a convergent subsequence of $\left(h_{1}^{\varepsilon}, h_{0}^{\varepsilon}\right)$ whose limit is denoted by $\left(h_{1}, h_{0}\right)$. On the other hand, it is easy to check that $\hat{y}_{0}^{\varepsilon} \rightarrow \hat{y}_{0}, \hat{x}_{0}^{\varepsilon} \rightarrow \hat{x}_{0}$ as $\varepsilon \rightarrow 0$. Thus (3.3) holds.

3.3. Maximum principle. In this subsection we derive the maximum principle for the case when $l(x, y, z, q, t)=0$ and $\phi(x)=0$. To this end, we introduce the adjoint process $(m(\cdot), n(\cdot))$ associated with the optimal solution $\xi^{0}$ to $(2.4)$, which is the solution of the following (forward) SDE:

$$
\left\{\begin{array}{l}
d m(t)=\left[\left(f_{x}^{0}(t)\right)^{*} m(t)+\left(g_{x}^{0}(t)\right)^{*} n(t)\right] d t+\left[\left(f_{q}^{0}(t)\right)^{*} m(t)+\left(g_{q}^{0}(t)\right)^{*} n(t)\right] d W(t) \\
m(0)=h_{1} \\
d n(t)=\left(g_{y}^{0}(t)\right)^{*} n(t) d t+\left(g_{z}^{0}(t)\right)^{*} n(t) d W(t) \\
n(0)=h_{0} \gamma_{y}\left(y^{0}(0)\right)
\end{array}\right.
$$

where $f_{x}^{0}(t), f_{q}^{0}(t), g_{x}^{0}(t), g_{y}^{0}(t), g_{z}^{0}(t), g_{q}^{0}(t)$ are as in $(3.1), y^{0}(0)=y_{0}^{\xi^{0}}$, and $h_{0}$ and $h_{1}$ are as in Theorem 3.2. It is easy to check that there is a unique process $(m(\cdot), n(\cdot))$ solving (3.5).

Define the Hamiltonian $H: R^{n} \times R^{m} \times L\left(R^{d} ; R^{m}\right) \times L\left(R^{d} ; R^{n}\right) \times R^{n} \times R^{m} \times[0, T] \rightarrow$ $R$ by

$$
H(x, y, z, q, m, n, t)=\langle m, f(x, q, t)\rangle+\langle n, g(x, y, z, q, t)\rangle
$$

We can rewrite the adjoint equation (3.5) in terms of the Hamiltonian as

$$
\left\{\begin{array}{l}
d m(t)=H_{x} d t+H_{q} d W(t) \\
m(0)=h_{1} \\
d n(t)=H_{y} d t+H_{z} d W(t) \\
n(0)=h_{0} \gamma_{y}\left(y^{0}(0)\right)
\end{array}\right.
$$

TheOrem 3.3. We assume (H1)-(H4). If $\xi^{0}$ is optimal to (2.4) with $\left(x^{0}(\cdot), y^{0}(\cdot)\right.$, $\left.q^{0}(\cdot), z^{0}(\cdot)\right)$ being the corresponding state of (2.3), then there exist $h_{1} \in R^{n}$ and $h_{0} \in R$ with $h_{0} \geq 0$ and $\left|h_{0}\right|+\left|h_{1}\right| \neq 0$ such that

$$
\left\langle m(T)+\left(h_{x}\left(\xi^{0}\right)\right)^{*} n(T), v-\xi^{0}\right\rangle \geq 0, \quad \text { a.s. }, \quad \forall v \in K,
$$

where $(m(\cdot), n(\cdot))$ is the solution of the adjoint equation (3.5).

Proof. For any $\xi \in U$ let $(\hat{x}(\cdot), \hat{y}(\cdot), \hat{q}(\cdot), \hat{z}(\cdot))$ be the solution to (3.1). Applying 
Itô's lemma to $\langle m(t), \hat{x}(t)\rangle+\langle n(t), \hat{y}(t)\rangle$ yields

$$
\begin{aligned}
& d[\langle m(t), \hat{x}(t)\rangle+\langle n(t), \hat{y}(t)\rangle] \\
= & {\left[\left\langle\hat{x}(t),\left(f_{x}^{0}(t)\right)^{*} m(t)\right\rangle-\left\langle m(t), f_{x}^{0}(t) \hat{x}(t)+f_{q}^{0}(t) \hat{q}(t)\right\rangle\right.} \\
& \left.+\left\langle\hat{q}(t),\left(f_{q}^{0}(t)\right)^{*} m(t)+\left(g_{q}^{0}(t)\right)^{*} n(t)\right\rangle\right] d t \\
& +\left[\left\langle\hat{y}(t),\left(g_{y}^{0}(t)\right)^{*} n(t)\right\rangle-\left\langle n(t), g_{x}^{0}(t) \hat{x}(t)+g_{y}^{0}(t) \hat{y}(t)\right.\right. \\
& \left.\left.+g_{z}^{0}(t) \hat{z}(t)+g_{q}^{0}(t) \hat{q}(t)\right\rangle+\left\langle\hat{z}(t), g_{z}^{0}(t) n(t)\right\rangle\right] d t \\
& +\{\ldots\} d W(t) \\
= & \{\ldots\} d W(t) .
\end{aligned}
$$

Integrating from 0 to $T$ and taking expectation, we obtain

$$
E\left[\left\langle m(T)+\left(h_{x}\left(\xi^{0}\right)\right)^{*} n(T), \xi-\xi^{0}\right\rangle\right]=\left\langle h_{1}, \hat{x}_{0}\right\rangle+h_{0}\left\langle\gamma_{y}\left(y^{0}(0)\right), \hat{y}_{0}\right\rangle \geq 0,
$$

where the inequality is due to (3.3). Since $\xi \in U$ is arbitrary, a standard argument yields (3.7). The proof is complete.

Denote the boundary of $K$ by $\partial K$. Set

$$
\Omega_{0} \triangleq\left\{\omega \in \Omega \mid \xi^{0}(\omega) \in \partial K\right\}
$$

By the above theorem, it is easy to see the following corollary holds.

Corollary 3.4. Under the assumptions of Theorem 3.3, for each $v \in K$,

$$
\begin{aligned}
& \left\langle m(T)+\left(h_{x}\left(\xi^{0}\right)\right)^{*} n(T), v-\xi^{0}\right\rangle \geq 0 \text { a.s. } \quad \text { on } \Omega_{0}, \\
& m(T)+\left(h_{x}\left(\xi^{0}\right)\right)^{*} n(T)=0 \text { a.s. } \quad \text { on } \Omega_{0}^{c} .
\end{aligned}
$$

3.4. The general case. In this subsection, we consider the general case where $l(x, y, z, q, t) \neq 0$ and $\phi(x) \neq 0$. Since the proof of the maximum principle in this case is essentially the same as in the preceding section, we only present the result without a proof.

Let $\xi^{0}$ be optimal to $(2.4)$ with $\left(x^{0}(\cdot), y^{0}(\cdot), q^{0}(\cdot), z^{0}(\cdot)\right)$ being the corresponding state of (2.3). We define the following adjoint equation analogous to (3.6):

$$
\left\{\begin{aligned}
d m(t)= & {\left[\left(f_{x}^{0}(t)\right)^{*} m(t)+\left(g_{x}^{0}(t)\right)^{*} n(t)+h_{0} l_{x}^{0}(t)\right] d t+\left[\left(f_{q}^{0}(t)\right)^{*} m(t)\right.} \\
& \left.\quad+\left(g_{q}^{0}(t)\right)^{*} n(t)+h_{0} l_{q}^{0}(t)\right] d W(t) \\
m(0)= & h_{1} \\
d n(t)= & {\left[\left(g_{y}^{0}(t)\right)^{*} n(t)+h_{0} l_{y}^{0}(t)\right] d t+\left[\left(g_{z}^{0}(t)\right)^{*} n(t)+h_{0} l_{z}^{0}(t)\right] d W(t) } \\
n(0)= & h_{0} \gamma_{y}\left(y^{0}(0)\right)
\end{aligned}\right.
$$

where $l_{k}^{0}(t)=l_{k}\left(x^{0}(t), y^{0}(t), z^{0}(t), q^{0}(t), t\right)$ for $k=x, y, z, q$.

TheOrem 3.5. We assume (H1)-(H4). If $\xi^{0}$ is optimal to (2.4) with $\left(x^{0}(\cdot), y^{0}(\cdot)\right.$, $\left.q^{0}(\cdot), z^{0}(\cdot)\right)$ being the corresponding state of (2.3), then there exist $h_{1} \in R^{n}$ and $h_{0} \in R$ 
with $h_{0} \geq 0$ and $\left|h_{0}\right|+\left|h_{1}\right| \neq 0$ such that

$$
\left\langle m(T)+\left(h_{x}\left(\xi^{0}\right)\right)^{*} n(T)+h_{0} \phi_{x}\left(\xi^{0}\right), v-\xi^{0}\right\rangle \geq 0 \quad \text { a.s. }, \quad \forall v \in K
$$

where $(m(\cdot), n(\cdot))$ is the solution of the adjoint equation (3.9).

CoROLlary 3.6. Under the assumptions of Theorem 3.5, for each $v \in K$,

$$
\begin{aligned}
& \left\langle m(T)+\left(h_{x}\left(\xi^{0}\right)\right)^{*} n(T)+h_{0} \phi_{x}\left(\xi^{0}\right), v-\xi^{0}\right\rangle \geq 0 \text { a.s. on } \Omega_{0}, \\
& m(T)+\left(h_{x}\left(\xi^{0}\right)\right)^{*} n(T)+h_{0} \phi_{x}\left(\xi^{0}\right)=0 \quad \text { a.s. } \quad \text { on } \Omega_{0}^{c} .
\end{aligned}
$$

\section{Applications.}

4.1. Stochastic LQ control with terminal state constraints. In this section, we study stochastic LQ optimal control problems with terminal state constraints. We consider two cases separately, depending on whether the state variable is a scalar or a vector.

4.1.1. One-dimensional case. Consider the following state equation:

$$
\left\{\begin{array}{l}
d x(t)=[A x(t)+u(t) B] d t+[C x(t)+u(t) D] d W(t), t \in[0, T], \\
x(0)=a,
\end{array}\right.
$$

where $A \in R^{1}, D \in R^{d \times d}, B \in R^{d}, C \in R^{1 \times d},(x(\cdot), u(\cdot)) \in M^{2}\left(0, T ; R^{1}\right) \times$ $M^{2}\left(0, T ; R^{1 \times d}\right)$ and $D^{-1}$ exists.

The set of admissible control is

$$
\mathcal{U}_{a d} \equiv\left\{u(\cdot) \mid u(\cdot) \in M^{2}\left(0, T ; R^{1 \times d}\right)\right\} .
$$

Without loss of generality we consider the cost function without a running cost:

$$
J(u(\cdot))=E\left[\frac{1}{2} x^{2}(T)\right] .
$$

Suppose the terminal constraint is $K=R^{+}$. Our optimization problem is to

$$
\begin{array}{ll}
\text { minimize } & J(u(\cdot)) \\
\text { subject to } & u(\cdot) \in \mathcal{U}_{a d} ; \quad x(T) \in K, \quad \text { a.s. }
\end{array}
$$

Problem (4.2) is a special case of (2.2) with

$$
\begin{aligned}
& b(x, u, t)=A x+u B ; \\
& \sigma(x, u, t)=C x+u D \\
& \phi(x)=\frac{1}{2} x^{2} \\
& \bar{g} \equiv 0, h \equiv 0, l \equiv 0, \gamma \equiv 0 .
\end{aligned}
$$

Now we give the backward formulation of (4.2). Setting $\bar{A}=A-C D^{-1} B$, $\bar{B}=D^{-1} B$ and $q(t) \equiv C x(t)+u(t) D$, we turn (4.1) into

$$
\left\{\begin{array}{l}
d x(t)=[\bar{A} x(t)+q(t) \bar{B}] d t+q(t) d W(t), t \in[0, T] \\
x(0)=a
\end{array}\right.
$$


Define

$$
U=\left\{\xi \mid \xi \in L^{2}\left(\Omega, \mathcal{F}_{T}, P\right), \xi(\omega) \in K, \text { a.s. }\right\}
$$

Then (4.2) can be rewritten as:

$$
\begin{array}{ll}
\text { Minimize } & J(\xi) \\
\text { subject to } & \xi \in U ; x^{\xi}(0)=a
\end{array}
$$

Define $\Omega_{0} \triangleq\left\{\omega \in \Omega \mid \xi^{0}(\omega)=0\right\}$ and the adjoint equation

$$
\left\{\begin{array}{l}
d m(t)=-\bar{A} m(t) d t-m(t) \bar{B}^{*} d W(t), \\
m(0)=h_{1}
\end{array}\right.
$$

where $h_{1} \in R$ is a parameter. Applying Theorem 3.5, we have

THEOREM 4.1. If $\left(x^{0}(\cdot), u^{0}(\cdot)\right)$ is optimal to (4.2), then there exist $h_{0}, h_{1} \in R$ with $h_{0} \geq 0$ and $\left|h_{0}\right|+\left|h_{1}\right| \neq 0$ so that $\xi^{0} \equiv x^{0}(T)$ satisfies

$$
\begin{array}{ll}
m(T)+h_{0} \xi^{0}=0 \text { a.s. } & \text { on } \Omega_{0}^{c}, \\
m(T)+h_{0} \xi^{0} \geq 0 \text { a.s. } & \text { on } \Omega_{0},
\end{array}
$$

where $m(\cdot)$ is solution to (4.3) with parameter $h_{1}$.

Proof. In this case, $f(x, q, t)=-[\bar{A} x+q \bar{B}], \phi(x)=\frac{1}{2} x^{2}$, and all the other functions are zero. So it is easy to verify the adjoint equation (3.9) specializes to (4.3). Since $K=R^{+}$, the necessary condition (3.11) becomes (4.4). The proof is complete.

COROLlary 4.2. The optimal $\xi^{0}=x^{0}(T)$ can be represented as

$$
\xi^{0}= \begin{cases}\left(-\frac{1}{h_{0}} m(T)\right)^{+}, & \text {if } h_{0}>0 \\ 0, & \text { if } h_{0}=0\end{cases}
$$

Proof. The result is clear if $h_{0}>0$ in view of the necessary condition given in Theorem 4.1. Now assume $h_{0}=0$, then $h_{1} \neq 0$. Hence

$$
m(T)=h_{1} \exp \left\{-\left[\bar{A}-\frac{1}{2}|\bar{B}|^{2}\right] T-\bar{B}^{*} W(T)\right\} \neq 0, \text { a.s. }
$$

However, the first equality in (4.4) yields $m(T)=0$ a.s. on $\Omega_{0}^{c}$; so $P\left(\Omega_{0}^{c}\right)=0$ or $\xi^{0}=0$ a.s.

The original problem (4.2) can now be solved completely in the following manner. First of all, Itô's formula derives that $m(\cdot) x^{0}(\cdot)$ is a martingale. Hence $E\left[m(T) x^{0}(T)\right]$ $=h_{1} a$. Now, if $a \leq 0$, then it is easy to see that the only $x^{0}(T)$ that satisfies Corollary 4.2 is $x^{0}(T)=0$. If $a>0$, then necessarily $h_{0}>0$ (otherwise the identity $E\left[m(T) x^{0}(T)\right]=h_{1} a$ will be violated) and $h_{1}<0$. In this case, the optimal state process $x^{0}(\cdot)$ is given by

$$
x^{0}(t)=m(t)^{-1} E\left(m(T) x^{0}(T) \mid \mathcal{F}_{t}\right)=-h_{0}^{-1} m(t)^{-1} E\left(m(T)^{2} \mid \mathcal{F}_{t}\right)
$$


An explicit expression of the above is possible as $m(T) m(t)^{-1}$ follows a lognormal distribution (with known parameters) conditional on $\mathcal{F}_{t}$, at any time $t$. Finally, once $x^{0}(\cdot)$ is obtained, the optimal control $u^{0}(\cdot)$ can be recovered by applying Itô's formula to $x^{0}(\cdot)$ and comparing its diffusion coefficient with the dynamics (4.1). We leave the details to interested readers.

To conclude this subsection, let us note that although the general result in the previous section is for a problem with only the terminal state constraint, for the current LQ case the result is also valid for pointwise (in time) state constraint. The essential reason is that any admissible state process whose terminal value is nonnegative keeps nonnegative all the time, as implied by the comparison theorem for BSDEs.

4.1.2. Multi-dimensional case. Consider the following state equation:

$$
\left\{\begin{array}{l}
d x(t)=[A x(t)+u(t) B] d t+u(t) d W(t), t \in[0, T], \\
x(0)=x_{0},
\end{array}\right.
$$

where $A \in R^{n \times n}, B \in R^{d},(x(\cdot), u(\cdot)) \in M^{2}\left(0, T ; R^{n}\right) \times M^{2}\left(0, T ; R^{n \times d}\right)$.

The set of admissible controls is

$$
\mathcal{U}_{a d} \equiv\left\{u(\cdot) \mid u(\cdot) \in M^{2}\left(0, T ; R^{n \times d}\right)\right\}
$$

and the quadratic cost function is

$$
J(u(\cdot))=E\left[\frac{1}{2}(x(T))^{*} Q x(T)\right]
$$

where $Q \in S^{n}$.

Suppose $K=\left(R^{n}\right)^{+}$. Similar analysis as with one-dimensional case leads to the following result.

TheOREM 4.3. If $\left(x^{0}(\cdot), u^{0}(\cdot)\right)$ is optimal, then there exist $h_{1} \in R^{n}$ and $h_{0} \in R$ with $h_{0} \geq 0$ and $\left|h_{0}\right|+\left|h_{1}\right| \neq 0$ so that $\xi^{0} \equiv x^{0}(T)$ satisfies

$$
\begin{array}{ll}
m(T)+h_{0} Q \xi^{0}=0 \text { a.s. } & \text { on } \Omega_{0}^{c}, \\
m(T)+h_{0} Q \xi^{0} \geq 0 \text { a.s. } & \text { on } \Omega_{0},
\end{array}
$$

where $m(\cdot)$ is the solution of the adjoint equation (4.6) below.

Proof. In this case,

$$
\begin{gathered}
f(x, q, t)=-[A x+q B], \\
\phi(x)=-\frac{1}{2} x^{*} Q x .
\end{gathered}
$$

The adjoint equation is

$$
\left\{\begin{array}{l}
d m(t)=-A^{*} m(t) d t-m(t) B^{*} d W(t), \\
m(0)=h_{1}
\end{array}\right.
$$

It follows from Theorem 3.5 that (4.5) holds. 


\subsection{A recursive optimization problem with bankruptcy prohibition.}

Consider a market where there are $d$ stocks and one bank account, and an investor who can decide at time $t \in[0, T]$ the amount $\pi_{i}(t)$ to invest in $i$ th stock $(i=1, \ldots, d)$ with initial wealth $a>0$. We assume the stock-volatility matrix $\sigma(\cdot)$ is a identity matrix to simplify our discussion. For more details about the financial setting, see [6]. Thus, in general, the wealth process $x(\cdot)$ satisfies the following equation

$$
\left\{\begin{array}{l}
d x(t)=b(x(t), \pi(t), t) d t+\pi(t) d W(t) \\
x(0)=a
\end{array}\right.
$$

where

$$
b(x, \pi, t): R^{1} \times R^{1 \times d} \times[0, T] \rightarrow R^{1} .
$$

An interesting example of such a nonlinear wealth equation is the optimal portfolio choice problem for a "large" investor considered in Cuoco and Cvitanic [4].

The recursive utility of the investor's terminal wealth $x(T)$ is described by the following BSDE:

$$
\left\{\begin{array}{l}
-d y(t)=g(y(t), z(t), t) d t-z(t) d W(t) \\
y(T)=h(x(T))
\end{array}\right.
$$

where

$$
\begin{aligned}
g(y, z, t) & : R^{1} \times R^{1 \times d} \times[0, T] \rightarrow R^{1}, \\
h(x) & : R^{+} \rightarrow R^{1} .
\end{aligned}
$$

We assume all coefficients satisfy (H1)-(H3). In addition, we assume

(H5) $\forall t \in[0, T], b(0,0, t) \geq 0$.

This hypothesis ensures by the comparison theorem that if the terminal wealth $x(T) \geq 0$, then the wealth process $x(\cdot)$ satisfies $x(t) \geq 0$ a.s., $0 \leq t \leq T$. Thus, under this hypothesis, no-bankruptcy prohibition is equivalent to terminal wealth constraint $x(T) \geq 0$ (see [7] and [3]).

Our problem is that under the bankruptcy prohibition, an investor chooses portfolio $\pi(\cdot)$ so as to maximize the recursive utility of his terminal wealth $x(T)$, i.e.,

$$
\begin{array}{ll}
\operatorname{maximize} & J(\pi(\cdot))=y(0) \\
\text { subject to } & \pi(\cdot) \in M^{2}\left(0, T ; R^{1 \times d}\right), \quad x(T) \geq 0, \quad \text { a.s. }
\end{array}
$$

It is easy to see that this is a special case of $(2.2)$ where $K=R^{+}$. Using the method in section 3 , we can obtain a stochastic maximum principle, i.e. a characterization of the optimal terminal wealth. 
Let $\xi^{0}$ be an optimal terminal wealth and $y^{0}(\cdot)$ and $x^{0}(\cdot)$ be the utility and the wealth processes associated with $\xi^{0}$. By Theorem 3.3, the adjoint processes are given by the processes $m(\cdot)$ and $n(\cdot)$ such that

$$
\left\{\begin{array}{l}
d m(t)=m(t)\left[f_{x}^{0}(t) d t+\left(f_{q}^{0}(t)\right)^{*} d W(t)\right] \\
m(0)=h_{1} \\
d n(t)=n(t)\left[g_{y}^{0}(t) d t+\left(g_{z}^{0}(t)\right)^{*} d W(t)\right] \\
n(0)=-h_{0}
\end{array}\right.
$$

where $f_{x}^{0}(t)=f_{x}\left(x^{0}(t), q^{0}(t), t\right), f_{q}^{0}(t)=f_{q}\left(x^{0}(t), q^{0}(t), t\right), g_{y}^{0}(t)=g_{y}\left(y^{0}(t), z^{0}(t), t\right)$, $g_{z}^{0}(t)=g_{z}\left(y^{0}(t), z^{0}(t), t\right)$. Note that in this case the mapping $\gamma(y)=-y$ which leads to $n(0)=-h_{0}$.

Set $\Omega_{0} \triangleq\left\{\omega \in \Omega \mid \xi^{0}(\omega)=0\right\}$ and suppose (H1)-(H3) and (H5). We have

THEOREM 4.4. There exist constants $h_{1}, h_{0} \in R$ with $h_{0} \geq 0$ and $\left|h_{1}\right|^{2}+\left|h_{0}\right|^{2} \neq 0$ such that

$$
\begin{array}{ll}
m(T)+n(T) h_{x}\left(\xi^{0}\right)=0 \text { a.s. } & \text { on } \Omega_{0}^{c}, \\
m(T)+n(T) h_{x}\left(\xi^{0}\right) \geq 0 \text { a.s. } & \text { on } \Omega_{0} .
\end{array}
$$

Remark: El.Karoui, Peng and Quenez [7] studied a general recursive utility optimization problem in which a consumption process was considered. Under convex (concave) assumptions, they used Lagrange multiplier method to derive a stochastic maximum principle which characterizes the optimal terminal state. Different from their approach, our method doesn't need convex (concave) assumptions.

5. Concluding Remarks. This paper investigates a state constrained stochastic control problem involving a forward-backward dynamics, and establishes a stochastic maximum principle via a backward formulation and Ekeland's principle. There are two main assumptions in this paper: 1) only the terminal state constraint is considered, and 2) the state constraint is a convex set. It remains an interesting and challenging open problem to weaken or completely remove these assumptions. 


\section{REFERENCES}

[1] M. Bardi And I. Capuzzo-Dolcetta, Optimal Control and Viscosity Solutions of HamiltonJacobi-Bellman Equations, Birkhäuser, Boston, 1997.

[2] L.D. Berkovitz, Optimal Control Theory, Springer-Verlag, New York, 1974.

[3] T. R. Bielecki, H. Jin, S. R. Pliska, And X. Y. Zhou, Continuous time mean variance portfolio selection with bankruptcy prohibition, Math. Finance, 15 (2005), pp. 213-244.

[4] D. Cuoco and J. Cvitanic, Optimal consumption choices for a "large" investor, J. Economic Dynamics and Control, 22 (1998), pp. 401-436.

[5] I. Ekeland, On the variational principle, J. Math. Anal. Appl., 47 (1974), pp. 324-353.

[6] N. El Karoui, S. Peng, And M. C. Quenez, Backward stochastic differential equations in finance, Math. Finance, 7(1997), pp. 1-71.

[7] N. El Karoui, S. Peng, And M.-C. Quenez, A dynamic maximum principle for the optimization of recursive utilities under constraints, Ann. Appl. Prob., 11(2001), pp. 664-693.

[8] S. JI, A Stochastic Optimization Problem with State Constraints and Its Applications in Finance, Ph. D. Thesis, Shandong University, 1999.

[9] S. Ji And S. PEng, Terminal perturbation method for the backward approach to continuous-time mean-variance portfolio selection, preprint, 2005.

[10] S. Peng, Backward stochastic differential equation and application to optimal control, Applied Mathematics and Optimization, 27 (1993), pp. 125-144.

[11] S. R. PlisKa, A discrete time stochastic decision model, Advances in Filtering and Optimal Stochastic Control, edited by W.H. Fleming and L.G. Gorostiza, Lecture Notes in Control and Information Sciences, 42, Springer-Verlag, New York, 290-304, 1982.

[12] S. R. PLISKA, Introduction to Mathematical Finance, Blackwell, Oxford, 1997.

[13] J. Yong And X. Y. Zhou, Stochastic Controls: Hamiltonian Systems and HJB Equations, Springer, New York, 1999. 